\title{
Acute upper limb ischemia in a patient with newly diagnosed paroxysmal atrial fibrillation
}

\author{
Dong Shin Kim, Seunghwan Kim, Hyang Ki Min, Chiwoo Song, Young Bin Kim, Sae Jong Kim, \\ Ji Young Park, Sung Kee Ryu, Jae Woong Choi \\ Department of Cardiology, Seoul Eulji Hospital, Eulji University College of Medicine, Seoul, Korea
}

\begin{abstract}
Acute limb ischemia (ALI) due to an embolism is associated with high mortality rate and poor prognosis, and early diagnosis with prompt revascularization is required to reduce the risk of limb amputation or even death. The etiologies of ALI are diverse, and it includes an embolism from the heart and thrombotic occlusion of the atherosclerotic native vessels, stents, or grafts. An uncommon cause of ALI is acute arterial thromboembolism, and atrial fibrillation (AF) is the single most important risk factors for systemic thromboembolism. It is important to correctly identify the source of ALI for secondary prevention, as it depends on the underlying cause. Percutaneous transluminal angioplasty (PTA) has been proven to be a safe and effective treatment for focal atherosclerotic and thrombotic occlusive diseases of the aorta and its major extremity branches. Herein, we report on a 77-year-old female patient with acute upper limb ischemia, treated by PTA using a catheter-guided thrombectomy. He was newly diagnosed with paroxysmal AF (PAF) while evaluation the cause of his acute arterial thromboembolism. We recommend that cardiologists always consider PAF as a possible diagnosis even in patients without any history of AF under ALI because it is possible to develop thromboembolism in clinical practice.
\end{abstract}

Keywords: Thromboembolism; Atrial fibrillation; Peripheral arterial disease; Angioplasty

\section{INTRODUCTION}

Acute limb ischemia (ALI) occurs as a result of sudden cessation of blood supply to an extremity threatening the viability of the affected limb. Major causes include thrombosis of a limb artery or bypass graft, embolism from the heart or a diseased artery, dissection, and trauma [1,2]. Atrial fibrillation $(\mathrm{AF})$ is the most common cardiac rhythm disturbance, which is predisposed to thrombus formation followed by a

Received: June 28, 2016, Revised: August 8, 2016 Accepted: August 16, 2016

Corresponding Author: Seunghwan Kim, Department of Cardiology, Seoul Eulji Hospital, Eulji University College of Medicine, 68 Hangeulbiseok-ro, Nowon-gu, Seoul 01830, Korea

Tel: +82-2-970-8671, Fax: +82-2-970-8429

E-mail: cloudksh@gmail.com risk for systemic thromboembolism that may leads to not only ischemic stroke but also to non-central nervous system (CNS) embolism, which contributes to increased morbidity and mortality [3,4]. Herein, we report a patient with acute upper limb ischemia treated by percutaneous transluminal angioplasty (PTA) using a catheter-guided thrombectomy, He was newly diagnosed with paroxysmal $\mathrm{AF}(\mathrm{PAF})$ during the evaluation process of the source of acute arterial thromboembolism. This may be particularly important when considering the various causes in patients with ALI from thromboembolic occlusion, in which PAF may play crucial role.

\section{CASE}

A 77-year-old female patient visited our emergency room presenting with severe persisting pain and numbness on the

Copyright (C) 2017 Yeungnam University College of Medicine

This is an Open Access article distributed under the terms of the Creative Commons Attribution Non-Commercial License (http://creativecommons.org/licenses/by-nc/4.0/) which permits unrestricted non-commercial use, distribution, and reproduction in any medium, provided the original work is properly cited. 
right hand for 3 hours. She had been treated for hypertension, and no other risk factors for cardiovascular diseases were present. An electrocardiogram on admission showed sinus bradycardia without ST segment and T wave abnormality. On physical examination, the right arm was hypothermic with cyanotic hand, and the upper right brachial arterial pulse was strong. However, the right radial arterial pulse was weak and the right ulnar arterial pulse was not palpable. Under im- pression of ALI, computed tomography (CT) angiography of the upper extremities revealed a total occlusion of the distal right brachial artery with distal run-off flow of the radial and ulnar arteries (Fig. 1). An emergent peripheral angiography was performed. We punctured two sites - upper brachial artery and radial artery - and inserted a 6 Fr sheath through the radial artery and baseline angiography via the upper brachial artery (Fig. 2A) and radial artery (Fig. 2B). The angiogra-
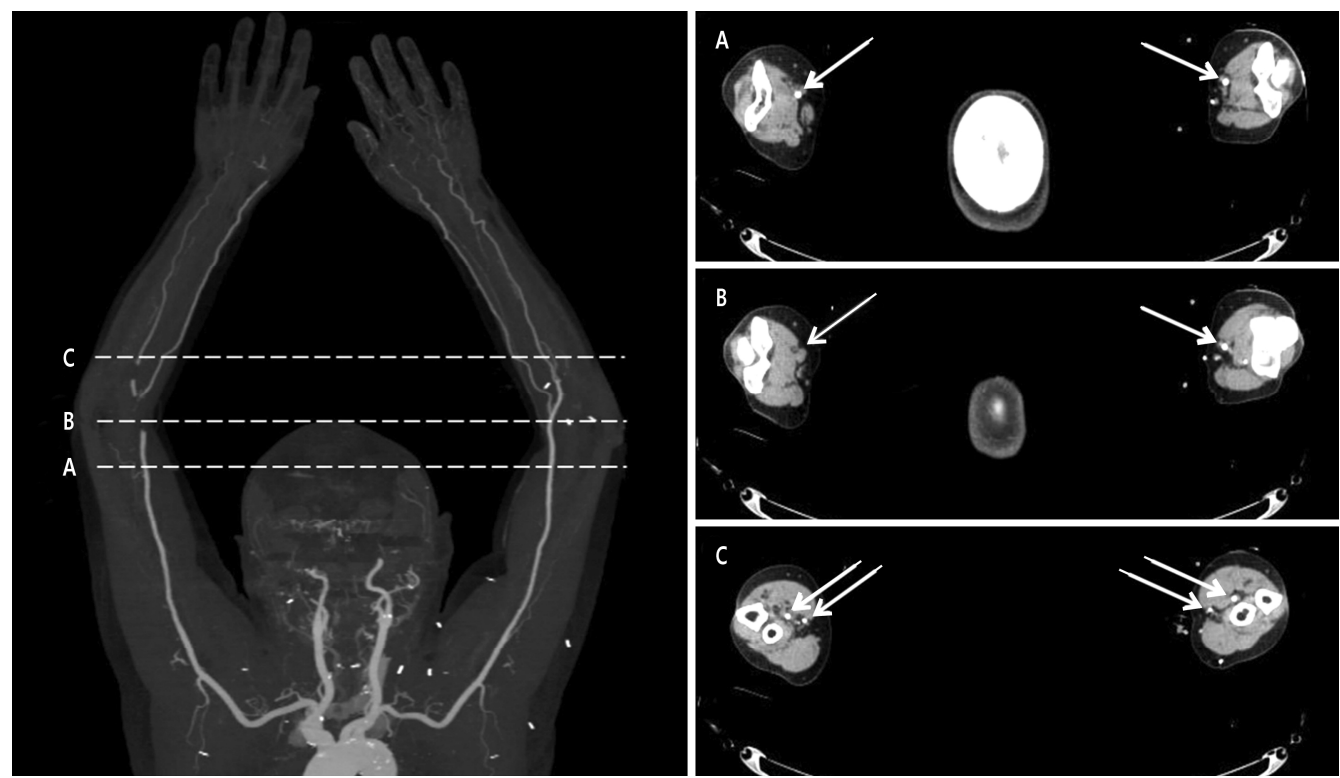

Fig. 1. Computed tomographic angiography of the upper-extremities showing total occlusion of the distal right brachial artery with a distal run-off flow of the radial and ulnar arteries (arrows).
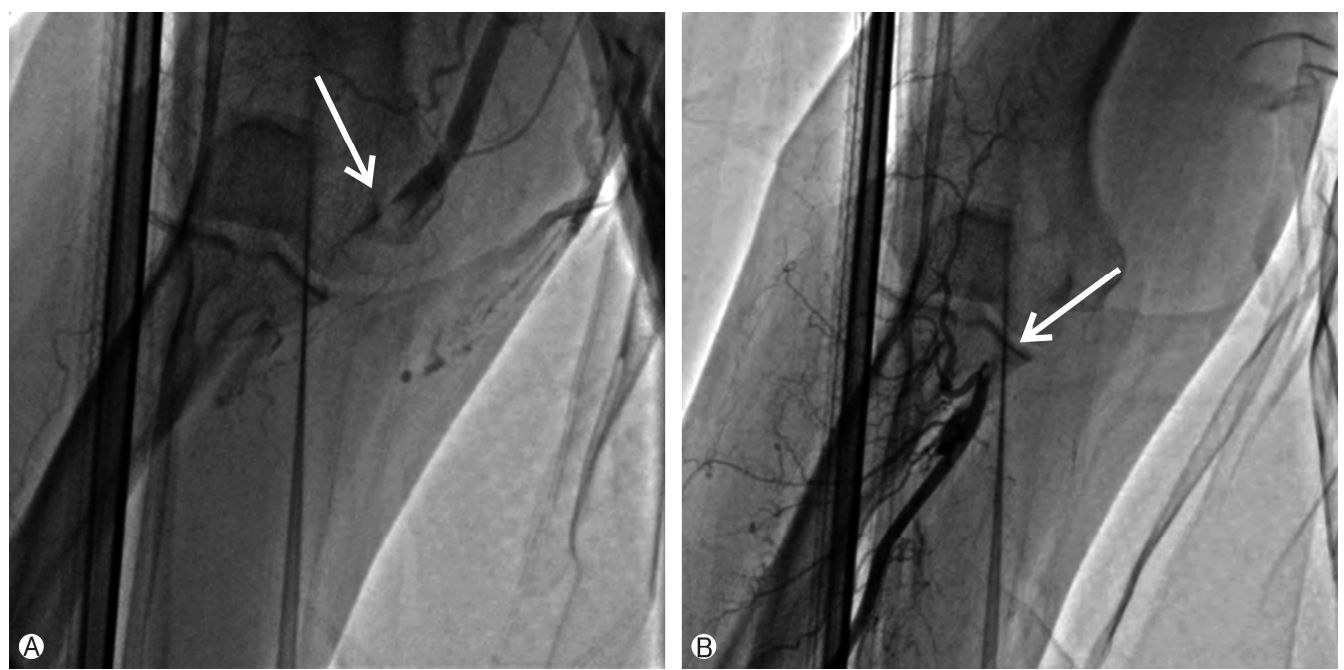

Fig. 2. Baseline angiography via the upper brachial artery (A) and radial artery (B) showing complete thromboembolic occlusion of the right brachial artery and bifurcation portion of the radial and ulnar arteries (arrows). 
phy showed complete thromboembolic occlusion at the right brachial artery and bifurcation portion of the radial and ulnar arteries. After confirming arterial occlusion of the upper extremity using an angiography, the right radial artery was first targeted for recanalization due to the dual supply of the hand and to prevent distal embolization. At first, ENVOY MPD guiding catheter (6 Fr, Johnson \& Johnson Medical Ltd, Pinewood Campus, Wokingham, UK) was advanced several times through the radial sheath retrogradely into the brachial artery for thrombosuction (Fig. 3A). After removing the large thrombus particles, we were able to observe the blood flow from the right brachial artery to the segments of the distal right radial and ulnar arteries, however remnant thrombi remained in the ulnar artery (Fig. 3B). Therefore, we advanced the ENVOY MPD guiding catheter through the brachial sheath antegradely into the ulnar artery for thrombosuction, several times (Fig. 3C, 3D). The final angiography revealed well visualized brachial artery, radial artery and ulnar artery with improved flow without stenosis (Fig. 3E). The patient's symp- toms disappeared. After PTA, using a catheter-guided thrombectomy, we evaluated the various examinations to clarify the causes of thromboembolism. In checking the hypercoagulable status, her profiles of the coagulation test were all within the reference range, and the anticardiolipin and lupus anticoagulant antibodies were negative. While investigating the causes of thromboembolism on the brachial artery, transthoracic echocardiography showed normal-sized cardiac chambers with normal left ventricular systolic function, Moreover according to the transesophageal echocardiography, there was no evidence of cardiac embolic sources. There was no evidence of a narrowed artery related with systemic atherosclerosis in the magnetic resonance angiography (MRA) and CT angiography. Although her initial electrocardiography showed normal sinus rhythm (Fig. 4A), we finally documented PAF on 24-hour Holter monitoring during admission (Fig. 4B). The cause of ischemic arm proved to be an embolic episode by PAF. Anticoagulation was started immediately after eliminating the emboli, which was soon changed to warfarin with
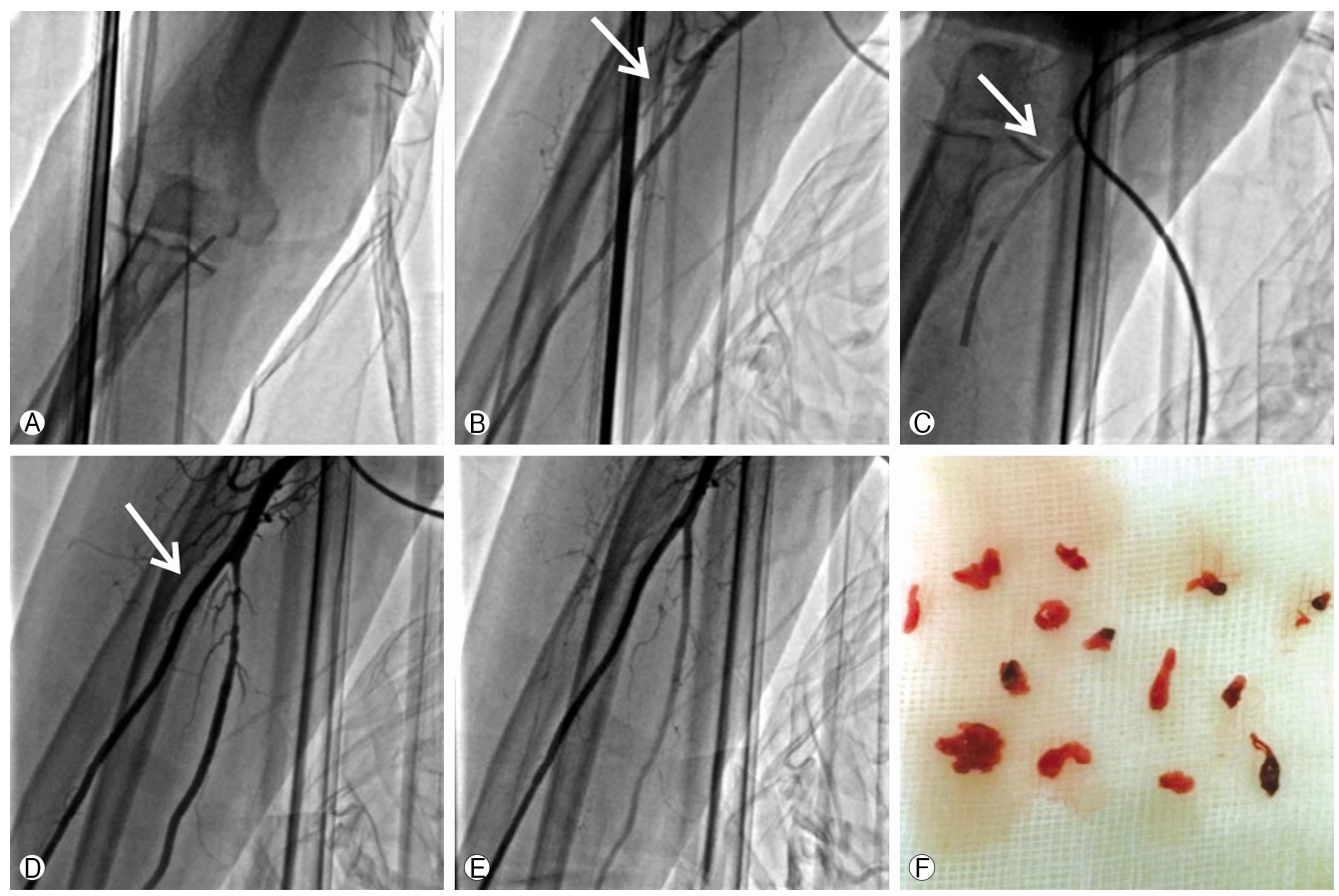

Fig. 3. (A) A 6 Fr guiding catheter was advanced through the radial sheath retrogradely into the brachial artery for thrombosuction. (B) After removal of large thrombus particles, blood flow could be seen from the right brachial artery to the segments of the distal right radial and ulnar arteries, but remnant thrombi were remained in the ulnar artery (arrow). (C, D) We advanced the suction catheter through the brachial sheath antegradely into the ulnar artery for thrombosuction, several times (arrows). (E) The final angiography after percutaneous aspiration thromboembolectomy revealed a well visualized brachial artery, radial artery, and ulnar artery with improved flow and no stenosis. (F) Large thrombus particles were removed. 


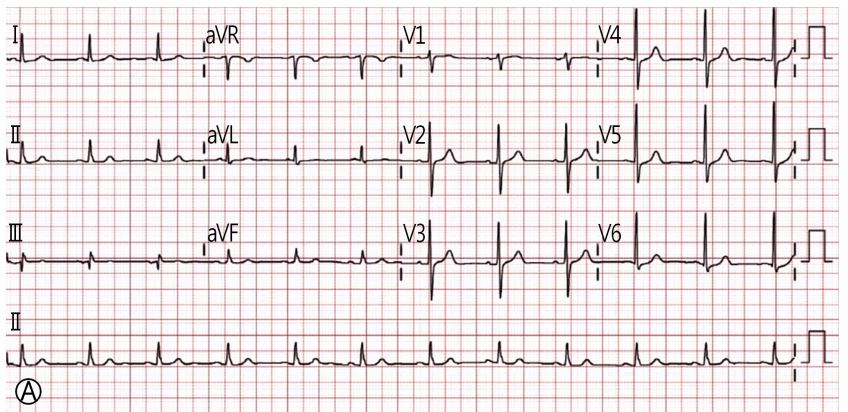

Fig. 4. (A) Initial electrocardiography showed normal sinus rhythm. documented.

target international normalized ratio between 2 and 3 . The patient has been treated with warfarin to prevent secondary embolic episodes.

\section{DISCUSSION}

ALI is defined as an abrupt interruption of blood flow to an extremity that threatens the viability of the limb, and it usually occurs due to either an embolic or thrombotic vascular occlusion [1]. It is different from chronic limb ischemia, in which the collateral vessels circumvent an occluded artery and compensate for the loss of perfusion, Conversely, ALI threatens limb viability because there is insufficient time for the growth of new blood-vessels to compensate [2]. This is a serious condition that is associated with high mortality rate and poor prognosis, requiring early recognition with prompt revascularization to reduce the risk of limb amputation [5].

Although acute thrombosis of the limb artery is most likely to occur at the site of an atherosclerotic plaque, limb-arterial thrombosis from cardiac embolism is a well-recognized but uncommon complication. AF is the most commonly sustained cardiac arrhythmia that has been increasingly observed in the elderly population and is associated with many possible complications, such as ischemic stroke, systemic thromboembolism, and heart failure [3,4]. In a Danish nationwide population-based study, 1,377 upper limb thromboembolectomies were reported, with an incidence rate of 3.3 and 5.2 per 100,000 person-years among men and women, respectively. The study showed that AF was the single most important risk factor for upper limb thromboembolectomy. They found that the risk factors were particularly high in women, which were associated with age, hypertension, myocardial infarction (MI), heart failure, and stroke [6]. Another systematic

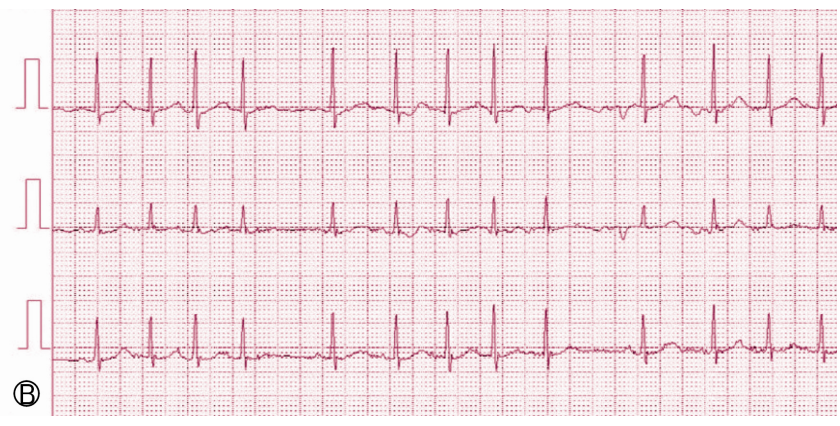

(B) On Holter monitoring, paroxysmal atrial fibrillation was finally review also found that age, female sex, $\mathrm{AF}$, hypertension, diabetes, MI, heart failure, and stroke were most common risk factors for the upper limb thromboembolectomies [5].

Most patients with arterial thromboembolism have AF, and the incidence of systemic embolism is not as common as that of stroke in patients with AF [7,8]. Moreover, thromboembolism of the upper limb is not as common as that of the lower limb. Therefore, the focus has been much more on the relationship with ischemic stroke rather than with peripheral thromboembolism.

A recent study showed that non-PAF (NPAF) is associated with a significantly increased risk of thromboembolism and death compared with PAF [9]. The risk of stroke and thromboembolism has been considered to be independent in between PAF and NPAF [10,11], and PAF is known to progress to NPAF in many patients over time. These results imply that patients with PAF should be treated similarly to NPAF [11].

Warfarin for stroke prevention in patients with non-valvular AF has been well documented. It has been shown in a recent meta-analysis that it was better than placebo and antiplatelet treatments with respect to the prevention of non-CNS systemic embolism, reducing the risk of systemic embolism by $71 \%$ as compared with placebo and by $50 \%$ as compared with antiplatelet therapy [12]. The availability of new oral anticoagulants, including thrombin inhibitors and factor $\mathrm{Xa}$ inhibitors, is promising for reducing the risk of systemic thromboembolism, and the benefit has been illustrated with respect to the prevention of stroke and systemic embolism $[7,8,13]$. In this case, the calculated level of the baseline creatinine clearance in accordance with the Cockcroft-Gault method was less than $30 \mathrm{~mL}$ per minute, we did not consider new oral anticoagulants.

The case of acute upper limb thromboembolism as the first 
manifestation of new onset PAF is very rare; however, if left untreated, it can leave severe sequelae. In this case, we checked the electrocardiography on admission, showing normal sinus rhythm. There was no evidence of cardiac embolic sources in the transthoracic and transesophageal echocardiography and no evidence of narrowed arteries related with systemic atherosclerosis in MRA and CT angiography. However, the 24-hour Holter monitoring during admission finally showed documented PAF. The first point of interest in this case is to perform an emergency endovascular revascularization, using a catheter-guided thrombectomy, which induced a dramatic result to restore blood flow as rapidly as possible without threatening tissue loss and limb viability. This shortened the duration of therapy. The second point of interest is making an effort to find the cause of thromboembolism on the upper extremities, and PAF was finally diagnosed, determining that the secondary prevention method in arterial occlusion had to be anticoagulation, not antiplatelet agents.

To the best of our knowledge, there have been only a few reported cases of acute arterial thromboembolism on the upper limb, undergoing treatment of endovascular intervention with catheter-guided thrombectomy, whereby the patient was newly diagnosed with PAF while evaluating the cause of acute arterial thromboembolism.

\section{CONFLICT OF INTEREST}

No potential conflict of interest relevant to this article was reported.

\section{REFERENCES}

1. Norgren L, Hiatt WR, Dormandy JA, Nehler MR, Harris KA, Fowkes FG, et al. Inter-society consensus for the management of peripheral arterial disease (TASC II). J Vasc Surg 2007; 45(Suppl S):S5-67.

2. Creager MA, Kaufman JA, Conte MS. Clinical practice. Acute limb ischemia. N Engl J Med 2012;366:2198-206.

3. Fuster V, Rydén LE, Cannom DS, Crijns HJ, Curtis AB, Ellenbogen KA, et al. ACC/AHA/ESC 2006 guidelines for the management of patients with atrial fibrillation: a report of the
American College of Cardiology/American Heart Association Task Force on Practice Guidelines and the European Society of Cardiology Committee for Practice Guidelines (Writing Committee to Revise the 2001 Guidelines for the Management of Patients With Atrial Fibrillation): developed in collaboration with the European Heart Rhythm Association and the Heart Rhythm Society. Circulation 2006;114:e257-354.

4. Nieuwlaat R, Prins MH, Le Heuzey JY, Vardas PE, Aliot E, Santini M, et al. Prognosis, disease progression, and treatment of atrial fibrillation patients during 1 year: follow-up of the Euro Heart Survey on atrial fibrillation. Eur Heart J 2008;29: 1181-9.

5. Andersen LV, Lip GY, Lindholt JS, Frost L. Upper limb arterial thromboembolism: a systematic review on incidence, risk factors, and prognosis, including a meta-analysis of risk-modifying drugs. J Thromb Haemost 2013;11:836-44.

6. Andersen LV, Mortensen LS, Lindholt JS, Faergeman O, Henneberg EW, Frost L. Upper-limb thrombo-embolectomy: national cohort study in Denmark. Eur J Vasc Endovasc Surg 2010;40:628-34.

7. Connolly SJ, Ezekowitz MD, Yusuf S, Eikelboom J, Oldgren $\mathrm{J}$, Parekh A, et al. Dabigatran versus warfarin in patients with atrial fibrillation. N Engl J Med 2009;361:1139-51.

8. Connolly SJ, Eikelboom J, Joyner C, Diener HC, Hart R, Golitsyn S, et al. Apixaban in patients with atrial fibrillation. N Engl J Med 2011;364:806-17.

9. Ganesan AN, Chew DP, Hartshorne T, Selvanayagam JB, Aylward PE, Sanders P, et al. The impact of atrial fibrillation type on the risk of thromboembolism, mortality, and bleeding: a systematic review and meta-analysis. Eur Heart J 2016;37: 1591-602.

10. Hart RG, Pearce LA, Rothbart RM, McAnulty JH, Asinger RW, Halperin JL. Stroke with intermittent atrial fibrillation: incidence and predictors during aspirin therapy. J Am Coll Cardiol 2000;35:183-7.

11. Hohnloser SH, Pajitnev D, Pogue J, Healey JS, Pfeffer MA, Yusuf $S$, et al. Incidence of stroke in paroxysmal versus sustained atrial fibrillation in patients taking oral anticoagulation or combined antiplatelet therapy: an ACTIVE W Substudy. J Am Coll Cardiol 2007;50:2156-61.

12. Andersen LV, Vestergaard P, Deichgraeber P, Lindholt JS, Mortensen LS, Frost L. Warfarin for the prevention of systemic embolism in patients with non-valvular atrial fibrillation: a meta-analysis. Heart 2008;94:1607-13.

13. Geller BJ, Giugliano RP, Braunwald E, Murphy SA, Hanyok JJ, Jin J, et al. Systemic, noncerebral, arterial embolism in 21,105 patients with atrial fibrillation randomized to edoxaban or warfarin: results from the Effective Anticoagulation With Factor Xa Next Generation in Atrial Fibrillation-Thrombolysis in Myocardial Infarction Study 48 trial. Am Heart J 2015;170:669-74. 